\title{
Understanidng Mobile Work Continuance of Chinese Knowledge Workers
}

\author{
Leida Chen \\ California Polytechnic State University \\ Lchen24@calpoly.edu
}

\begin{abstract}
As more and more organizations adopt a "mobile by default" approach to information systems and work design, studying mobile work as a post-adoption phenomenon is both important and timely. Using data collected from 238 Chinese mobile workers, this study develops and validates a model of mobile work continuance. Our findings suggest that the expectation-confirmation framework provides strong theoretical support for explaining mobile work continuance, and that performance, technical support, management support, data security concerns and work life balance concerns affect knowledge workers' mobile work continuance intention collectively.
\end{abstract}

\section{Introduction}

Despite the wide adoption of mobile work technologies and practices, few organizations have shown concerted efforts to assess and manage mobile work acceptance and continuance. Consequently, many organizations have not been able to reap the full benefits of their investments in this area [12]. This can be partially attributed to the fact that explosive adoption of mobile work technologies and practices by business professionals has severely outpaced research efforts in this field [32]. The number of empirical studies that focus on increasing employee acceptance and continuance of mobile work technologies and practices is especially limited $[4,52]$. Adopting the theory of information systems (IS) continuance, this study aims to explain mobile work continuance and identify the antecedents of mobile work continuance intention of knowledge workers. Up to date, very few studies focus on IS continuance behaviors of users compared to the number of studies on IS acceptance (e.g. $[16,51])$. However, the motivation to continue to use a system is arguably as important as, if not more important than, factors that influence the user's initial acceptance of the system [5]. Today, mobile work has become the norm in corporate scenes, and more and more organizations have adopted a "mobile by default" approach to IS and work design. Therefore, studying mobile work as a post-adoption phenomenon is both important and timely. Following this rationale, this paper posits that understanding mobile work continuance is crucial to the broader adoption of and improving organizations' return on investments in mobile work technologies and practices. By expanding the expectation-confirmation theory (ECT) of IS continuance, this study examines the impact of work outcome and experiential constructs on employees' decision to continue adopting mobile work technologies and practices.

\section{Literature Review}

Mobile work is enabled by mobile devices and services that extend access to work information and powerful computing capacity beyond the office to offer organizations and their employees with real-time information access and processing [45]. The trend of digital convergence helped organizations reduce their communications costs significantly and, at the same time, centralize all forms of communications between employees, enterprise, and customers. Mobile work has been found to offer greater flexibility, convenience, increased personal empowerment, higher quality of work life, real estate savings, productivity gains, enhanced customer services, the ability to blend expertise across space, and employee retention and satisfaction $[11,14,19,28,41]$. The positive impact of mobile work on organizational performance was validated by a recent study [45] which found that organizational performance enhancement could be explained by the positive impact of mobile business on sales and marketing and internal operations, two areas where mobile work was frequently performed.

However, mobile work is not without risks to organizations and their employees. One of the widely discussed technical threats is data and information security. Studies revealed that a large percentage of mobile workers had not taken the necessary steps to protect their computers and data and that most executives ranked security as the number one obstacle for mobile work [21, 50]. Another key area of controversy is the notion of supervision in the mobile work context. Studies vary greatly on this point. Managers claim that they are having difficulty monitor employee activities, measuring productivity or ensuring task completion in a mobile work environment [27]. Other studies suggest that managers must resist the urge to evaluate mobile 
workers using simple measures of behaviors and appearance, such as how long the employee has been connected to the corporate system and how many clients the employee has contacted, and instead implement a "results-only work environment" which demolishes the decades-old business dogma that equates physical presence with productivity $[14,30]$.

Furthermore, mobile work tends to create overlaps between societal, work and personal contexts. As the result, a mobile worker's patterns of work become more episodic or intermittent and require rapid role switching between the individual's roles in work and non-work contexts $[15,18]$. A number of studies suggest that mobile work may infringe on employees' work-life boundaries [17, 29, 42, 47]. Specifically, Jarvenpaa et al. [29] described how anytime, anywhere work had become "all-the- time, everywhere work" for some mobile workers. The ease of access to information and people, made possible by new technologies, has raised organizations' expectation to receive immediate responses from their employees. The spillover from work to personal life will likely have long-term negative effects on employees' productivity $[13,17]$.

Corporate culture has been found to be a deterrent to the acceptance of mobile work [9, 27]. The impact of culture on new forms of work was recognized by the early research of Olson [44] and Gordon [25]. Olson in particular noted that the corporate need for "control" discouraged organizations from adopting technologies that support flexible work environments. A study by Hoang et al. [27] also found that in most companies, the culture was incompatible with mobile work due to its emphasis on visibility, face-to-face interaction, and the notion of "visibility is promotability". A more recent study offered evidence that a culture characterized by deep trust and flat management system works best to facilitate effective mobile work adoption [35].

A number of prior studies focused on identifying the factors that contributed to mobile work success. Adopting the socio-technical perspective, Chen and Nath's [12] study identified seven impediment factors of mobile work using a nation-wide survey of CIOs. The impediment factors include difficulty with mobile work support, behavior issues of mobile workers, data/information/network security concerns, difficulty with mobile connectivity, mobile worker isolation, lack of clarity on mobile-work/worker-technology fit and mobile worker management concerns. In addition, eleven strategies were identified and found to correlate significantly with self-reported mobile work success. Some prior studies focused on technology acceptance in the context of mobile work and found that traditional adoption theories such as technology acceptance model (TAM) and innovation diffusion theory explained and predicted user initial adoption of mobile systems [38]. Studies have found that compatibility between work and mobile work support systems was critical to user satisfaction and acceptance $[34,52]$. In addition, utility, social value, age and gender also played a role in user adoption of mobile technologies [33]. Nevertheless, employees' continuance intention of mobile work systems and practices was largely left unstudied to the best of our knowledge.

\section{Research Model and Hypotheses}

Social psychology theories such as theory of reasoned action (TRA) [22] and theory of planned behavior (TPB) [1] support the notion that individuals' behaviors are consistent with their behavioral intention. This notion is widely adopted by IS theories such as TAM and unified theory of acceptance and use of technology (UTAUT) and supported by empirical studies on IS use and continuance behaviors (e.g. [7, 16, 48]. Therefore, this study decides to focus on understanding individuals' continuance intention of mobile work as a proxy of their continuance behaviors. To explore the factors that contribute to the continuance intention of mobile work, this project draws upon the theoretical work of Bhattacherjee [5], which was the most widely adopted framework that conceptualized IS continuance and demonstrated the distinctions between acceptance and continuance behaviors. The model posits that IS continuance intention is primarily determined by users' satisfaction with their prior IS use. In other words, satisfied users tend to continue their use of the system, and vice versa. The model stipulates that user satisfaction is determined by users' expectation of the IS, which is represented as ex post perceived usefulness, and confirmation of expectation following actual use. Consistent with TAM, perceived usefulness is also a direct predictor of IS continuance intention. Finally, users' confirmation of expectation tends to affect usefulness perception in order to keep user expectation consistent with reality.

While the ECT model provides an excellent theoretical foundation, it has left out key constructs that are relevant to the mobile work context. Many researchers have advocated extending the model to better predict the continuance intention of users. For example, Chen et al.'s [10] study on IS continuance of information-oriented mobile applications (IOMA) puts forth the concepts of outcome-related and systemrelated constructs that served as antecedents of IOMA continuance. The conceptual model underlying the current research follows the same rationale to explain mobile work continuance by extending the ECT (see Figure 1, Model of Mobile Work Continuance). The 
model expands the post-acceptance model of IS continuance by including work outcome constructs (i.e. performance) and experiential constructs (i.e. organizational technology support, management support, data security concerns and work-life balance concerns) that were found to influence mobile work success in prior studies (e.g. [12]) but have not been examined in the context of mobile work continuance.

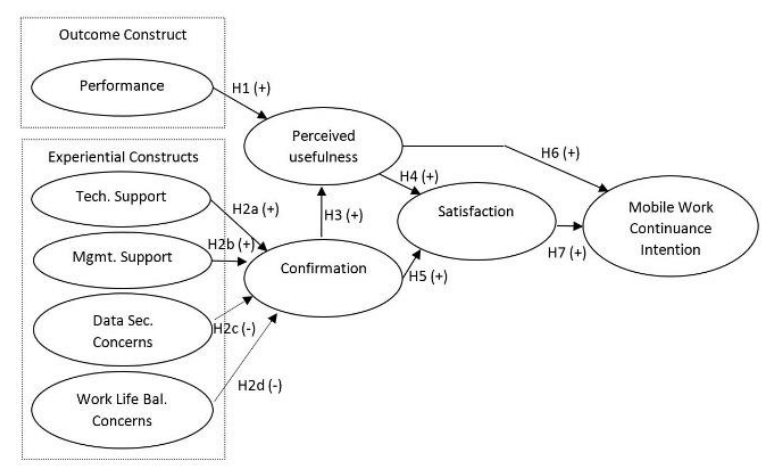

\section{Figure 1. Research Model of Mobile Work Continuance}

In the proposed model, perceived usefulness (PU) represents the user's post-usage (ex post) expectation of the IS following actual use. Performance enhancement is the primary desired system outcome, especially in the context of work-related systems. The enhancement or decline in performance experienced by the user as the result of adopting mobile work will likely raise or tone down the user's expectation of the usefulness of mobile work in the future [7]. Hence, we hypothesize:

H1 Perceived usefulness of continued mobile work is positively influenced by the performance enhancement they experience as the result of adopting mobile work (PER $\rightarrow$ PU).

While work outcome constructs are hypothesized to affect PU, experiential constructs are hypothesized to affect $\mathrm{C}$. While both antecedents of satisfaction (SAT), C and PU represent different cognitive levels. $\mathrm{C}$ refers to the user's realization of the expected experience of IS use. Prior empirical studies have shown that employee mobile work experience could be most significantly influenced by the following factors: organizational device and technology support (TECH), management support (MGMT), data security concerns (SEC) and work-life balance concerns (WLB) (e.g. [12]). TECH refers to the extent, timeliness and quality of technical support that mobile workers receive and mobile workers' satisfaction with the technical support provided. MGMT deals with organizations' efforts to provide management support, policies and culture that are conducive to mobile work. SEC refers to mobile workers' concerns about data security risks during mobile work. Finally, WLB deals with mobile workers' concerns regarding the spillover of work into personal life. The aforementioned experiential constructs are included in the research model and hypothesized to influence $\mathrm{C}$. The following hypotheses are proposed:

$\mathrm{H} 2 \mathrm{a}$ Confirmation of expectations of mobile work is positively influenced by the level of organizational device and technical support they receive as mobile workers (TECH $\rightarrow$ C).

$\mathrm{H} 2 \mathrm{~b}$ Confirmation of expectations of mobile work is positively influenced by the level of management support they receive as mobile workers (MGMT $\rightarrow \mathrm{C}$ ).

H2c Confirmation of expectations of mobile work is negatively influenced by their data security concerns related to mobile work $(\mathrm{SEC} \rightarrow \mathrm{C})$.

$\mathrm{H} 2 \mathrm{~d}$ Confirmation of expectations of mobile work is negatively influenced by their work life balance concerns related to mobile work (WLB $\rightarrow$ C).

Finally, the links in the original post-acceptance model of IS continuance are hypothesized in this study to confirm the validity of adopting the ECT framework in the mobile work context. Therefore, the following hypotheses are proposed:

H3 Perceived usefulness of continued mobile work is positively influenced by their confirmation of expectations of mobile work (C $\rightarrow$ PU).

H4 Satisfaction with mobile work is positively influenced by their perceived usefulness of continued mobile work (PU $\rightarrow$ SAT).

H5 Satisfaction with mobile work is positively influenced by their confirmation of expectations of mobile work $(\mathrm{C} \rightarrow \mathrm{SAT})$.

H6 Mobile work continuance intention is positively influenced by their perceived usefulness of continued mobile work (PU $\rightarrow \mathrm{CI})$.

H7 Mobile work continuance intention is positively influenced by their satisfaction with mobile work (SAT $\rightarrow \mathrm{CI})$.

\section{Research Methodology}


The items used to measure the constructs in the research model were drawn from previous studies that have measured the same constructs and modified to fit the context of mobile work. In cases where no prior measure was identified, new items were developed. Constructs, their definitions and sources of items used to measure the constructs are summarized in Table 1 . 42 items were included in the final questionnaire. The items were written in the form of statements and survey participants were asked to indicate to what extent they agreed or disagreed with the statement on a 5-point Likert scale. To ensure the full comprehension of the questionnaire by the intended samples of this study, Chinese knowledge workers, the items, originally designed in English, were professionally translated into Chinese. To verify that there were no translation biases, the items were backtranslated to English by a researcher who was not associated with this study. No significant distinctions were detected between the original items and the backtranslated version of the items suggesting the absence of translation biases in the final questionnaire.

\section{Table 1. Model Constructs}

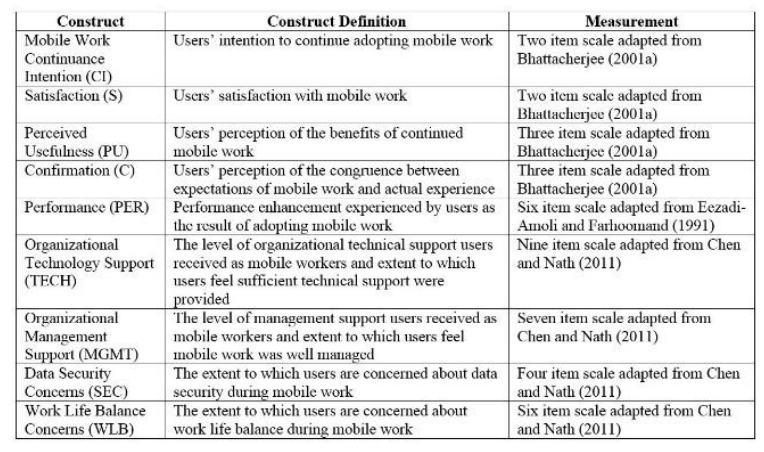

Data were collected using a survey of mobile workers from 30 organizations in two metropolitan areas in China. These organizations were identified to have adopted mobile work technologies and practices and offer their employees the choice of mobile and remote work. Senior managers from the organizations were recruited through personal and professional connections to help with the data collection process. An email that contained the link to the online survey used in this study was distributed by the senior managers to employees who engaged in mobile work practices at least one day a week in their respective organizations. The email also explained the purpose of the study and encouraged respondent participation by assuring the confidentiality of their responses. To control for the nature of respondents' job function, the senior managers were instructed to only send the survey to knowledge workers, who are defined as white collar, skilled workers who perform knowledge- rich jobs that involve handling and using information [46]. Therefore, clerical employees or workers whose jobs mostly involve manual labor were not included in this study. The responses to the survey were submitted directly to the researcher of this study to ensure confidentiality. A total of 1052 emails were distributed by the executives of the 30 companies, and 238 returned responses were found to be complete and usable for this study, rendering a response rate of $22.6 \%$. The relatively high response rate may be attributed to the endorsement and support of the executives of the participating organizations. Table 2 and 3 summarize the respondent and organization profiles. As the tables show, a wide range of industries, organization types and sizes, genders, ages, job functions and years of mobile work experience are represented in the data.

Table 2. Respondent Profiles

\begin{tabular}{|l|l|l|}
\hline & Frequency & $\begin{array}{l}\text { Percent } \\
(\%)\end{array}$ \\
\hline Gender & & \\
\hline Female & 132 & $55.5 \%$ \\
\hline Male & 106 & $44.5 \%$ \\
\hline Age & & \\
\hline below 25 & 38 & $16.0 \%$ \\
\hline $25-34$ & 72 & $30.3 \%$ \\
\hline $35-44$ & 67 & $28.2 \%$ \\
\hline $45-54$ & 36 & $15.1 \%$ \\
\hline 55 and over & 25 & $10.5 \%$ \\
\hline $\begin{array}{l}\text { Years of Mobile } \\
\text { Work }\end{array}$ & & \\
\hline$<1$ year & 45 & $18.9 \%$ \\
\hline $1-3$ years & 74 & $31.1 \%$ \\
\hline $3-5$ years & 63 & $26.5 \%$ \\
\hline Over 5 years & 56 & $23.5 \%$ \\
\hline Functional Areas & & \\
\hline Accounting & 26 & $10.9 \%$ \\
\hline Engineering & 16 & $6.7 \%$ \\
\hline Finance & 33 & $13.9 \%$ \\
\hline Human Resources & 19 & $8.0 \%$ \\
\hline $\begin{array}{l}\text { Information } \\
\text { Technology }\end{array}$ & 58 & $24.4 \%$ \\
\hline Legal & 7 & $2.9 \%$ \\
\hline Management/Admin. & 38 & $16.0 \%$ \\
\hline Marketing \& Sales & 36 & $15.1 \%$ \\
\hline Public Relations & 5 & $2.1 \%$ \\
\hline & & \\
\hline
\end{tabular}

Table 3. Organizational Profiles

\begin{tabular}{|l|l|l|}
\hline & Frequency & $\begin{array}{l}\text { Percent } \\
(\%)\end{array}$ \\
\hline Industry & & \\
\hline
\end{tabular}




\begin{tabular}{|l|l|l|}
\hline Advertising & 2 & $6.7 \%$ \\
\hline Consulting & 1 & $3.3 \%$ \\
\hline Education & 2 & $6.7 \%$ \\
\hline Energy & 2 & $6.7 \%$ \\
\hline Financial Services & 4 & $13.3 \%$ \\
\hline Healthcare & 1 & $3.3 \%$ \\
\hline Information Technology & 5 & $16.7 \%$ \\
\hline Manufacturing & 4 & $13.3 \%$ \\
\hline Pharmaceutical & 2 & $6.7 \%$ \\
\hline Real Estate & 4 & $13.3 \%$ \\
\hline Telecommunications & 3 & $10.0 \%$ \\
\hline $\begin{array}{l}\text { Company/Organization } \\
\text { Type }\end{array}$ & & \\
\hline Chinese Domestic & 18 & $60.0 \%$ \\
\hline Multinational & 12 & $40.0 \%$ \\
\hline & & \\
\hline Annual Revenue & & $23.3 \%$ \\
\hline $\begin{array}{l}\text { Below 500 Million Yuan } \\
\text { (Approx. US\$80 Million) }\end{array}$ & 7 & $63.3 \%$ \\
\hline $\begin{array}{l}\text { 500 Million Yuan to 10 } \\
\text { Billion Yuan (Approx. } \\
\text { US\$1.6 Billion) }\end{array}$ & 19 & $13.3 \%$ \\
\hline Over 10 Billion Yuan & 4 & \\
\hline
\end{tabular}

\section{Data Analysis}

The two-step structural equation modeling (SEM) approach recommended by Anderson and Gerbing [2] was employed for data analysis. In the first step, the reliability and validity of the factorial structure for the measurement model was assessed using confirmatory factor analysis (CFA). This allowed researchers to evaluate how well the questionnaire items measured the latent factors that they were designed to measure. The second step tested the causal structure proposed in the research model using the structural equation modeling technique. The technique allows simultaneous analysis of the relationships between the latent constructs and tests the hypotheses postulated by this study.

\subsection{Measurement Model}

CFA was performed on all the items simultaneously to evaluate the validity of the items and nine underlying constructs in the measurement model. The initial results suggested that some construct revisions were needed to improve the model fit. Items recommended for deletion were evaluated from both a statistical and a substantive point of view before deletion to ensure that the deletion of the items would not jeopardize the theoretical integrity of the constructs. Six items were ultimately deleted. The final measurement model was re-specified to include 36 items to measure the nine constructs of the research model. All items were found to have relatively high factor loadings (> 0.6) on the constructs they are measuring that are significant at the 0.01 level. The resulting measurement model demonstrates good fit with the empirical data. The normalized Chi-square (Chi-square / degrees of freedom) is 2.062, which is less than the recommended value of 3.0, suggesting good overall fit of the measurement model. Both Normalized Fit Index (0.907) and Comparative Fit Index (0.926) are greater than the benchmark value of 0.90 , and both RMSEA (0.06) and RMR (0.045) are within the range of acceptability recommended by MacCallum et al. [39] and Byrne [8].

The internal consistency of the measurement model was assessed by calculating the composite reliability coefficients, which are displayed in Table 4. All latent variables have reliability coefficients higher than the benchmark of 0.60 recommended by Bagozzi and Yi [3] suggesting the existence of high internal reliability in the measurement model. To assess the convergent validity, Average Variance Extracted (AVE) coefficients were computed and displayed in Table 4. All measures exceed the 0.5 benchmark as recommended by Fornell and Larcker [23] and Hair et al. [26]. In addition, all factor loadings were significant at the 0.01level and at 0.693 or above. Satisfactory composite reliability, AVEs and loadings jointly confirm the convergent reliability of our measurement model.

\section{Table 4. Composite Reliability and Average Variance Extracted}

\begin{tabular}{|l|l|l|}
\hline $\begin{array}{l}\text { Latent } \\
\text { Variables }\end{array}$ & $\begin{array}{l}\text { Composite } \\
\text { Reliability } \\
\text { (CR) }\end{array}$ & $\begin{array}{l}\text { Average Variance } \\
\text { Extracted (AVE) }\end{array}$ \\
\hline CI & 0.896 & 0.812 \\
\hline SAT & 0.870 & 0.769 \\
\hline PU & 0.899 & 0.748 \\
\hline C & 0.869 & 0.689 \\
\hline PER & 0.951 & 0.763 \\
\hline TECH & 0.946 & 0.713 \\
\hline MGMT & 0.900 & 0.644 \\
\hline SEC & 0.887 & 0.724 \\
\hline WLB & 0.902 & 0.649 \\
\hline
\end{tabular}

\subsection{Structural Model}

Using the SEM technique, the initial test of the research model showed reasonable fit between the model and empirical data. However, a review of the structural parameter estimates and modification 
indices suggests that some re-specifications of the structural model would yield improved model fit. The link between MGMT and C was not statistically significant; therefore, the structural path was subsequently removed from the model. Modification indices suggest two additional paths, MGMT $\rightarrow \mathrm{CI}$ and WLB $\rightarrow$ CI, would improve model fit. Prior studies have also found that variables other than PU and SAT could influence CI directly (e.g. [6, 10]), hence the research model was revised to include these two structural paths.

The estimations of the revised model yielded a Chi-square of 1254 with 569 degrees of freedom. The Chi-square/degrees of freedom ratio was 2.20 , with a CFI of 0.913 and NFI of 0.905 , suggesting good overall fit of the structural model. Both RMSEA (0.07) and RMR (0.05) are within the range of acceptability. Figure 2 displays a schematic representation of the resulting model with the structural coefficients and standard errors of the structural paths. All of the structure paths were statistically significant at the 0.01 or 0.001 level. Overall, the structural model demonstrates good fit with the empirical data.

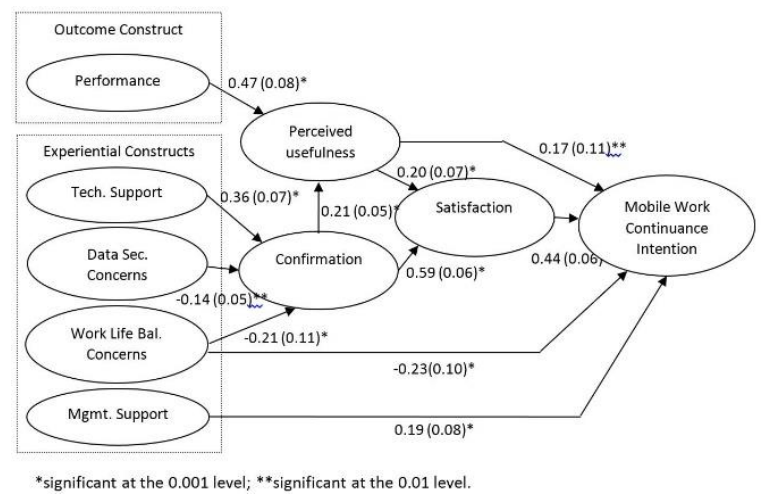

Figure 2. SEM Results of the Research Model

The hypotheses about the relationships between the constructs in the model were tested through the significance of the structural coefficients. All but one hypothesis are supported. H2b which hypothesizes the relationship between management support and confirmation (MGMT $\rightarrow$ C) was not supported by the data suggesting that managerial support received by mobile workers did not significantly influence their confirmation of pre-usage expectations. In addition to the previously stated hypotheses, the two new paths yielded statistically significant structural coefficients. The new paths suggest that managerial support and work life balance concerns influence mobile workers' continuance intention directly. All hypotheses related to the ECT model of IS continuance are supported verifying the validity of adopting the ECT framework in the mobile work context.

\section{Discussion and Implications}

Our findings suggest that the ECT theoretical framework is valid and useful in explaining mobile work continuance intention. As the result, all the hypotheses related to the original IS continuance model (hypotheses 3 through 7) were supported by the data. Mobile workers' continuance intention was found to be influenced by their affective response to mobile work experience (i.e. satisfaction), which was in turn influenced by the positive perception of continued mobile work benefits (i.e. perceived usefulness) and confirmation of the pre-usage expectations (i.e. confirmation). Consistent with the IS continuance model, this study also found that confirmation influenced perceived usefulness and that perceived usefulness impacted continuance intention directly. Furthermore, the study found that performance enhancement resulted from mobile work positively impacted perceived usefulness. This finding suggests that users' performance gain as the result of adopting mobile work has profound impact on the continuance decision as it helps set the tone for the perception of the benefits of continued adoption of mobile work. Since perceived usefulness was found to be a key determinant of users' satisfaction with and continuance intention of mobile work, whether mobile work was successful in enhancing one's performance becomes an important barometer for assessing users' continuance intention of engaging in mobile work. The positive performance - perceived usefulness behavior intention link suggests that mobile work continuance can be explained as a rational cognitive process motivated by the post-usage benefits of the system. At the same time, the positive performance perceived usefulness - satisfaction - continuance intention link suggests that enhanced work outcomes lead to positive affective response to the system which in turn positively reinforces the user's continuance intention.

Experiential constructs including organizational device and technology support, data security concerns and work life balance concerns were found to impact mobile work continuance decision through the confirmation of users' pre-usage expectations. These constructs gauge the experience and concerns of users when engaging in mobile work. As expected, organizational technology supported influences confirmation positively while data security and work life balance concerns influence confirmation negatively. Therefore, these findings suggest providing superior device and technology support to mobile workers and reducing their data security and 
work life balance concerns would collectively enhance users' mobile work experience and lead to a positive realization of the expected benefits of mobile work. When users' expectations regarding mobile work are met or exceeded, users develop positive affective response which in turn leads to continuance intention of mobile work. These findings suggest that experiential constructs are critical to users' formation of positive affective response to mobile work as they help confirm users' pre-usage expectations.

The research findings have implications for both theory and practice. From the theoretical perspective, this study makes a significant contribution to the understanding of mobile work continuance as there is currently a shortage of published research that investigates individuals' continuance behaviors in the mobile work context. This study confirms the validity of the ECT framework of IS continuance to explain a new domain, mobile work, and the validation suggests that the ECT framework may be applied with reasonable confidence to study the continuance intention of other IT-enabled work practices. In addition, this study enhances our understanding of mobile work continuance by identifying meaningful and actionable antecedents that help explain what makes mobile workers continue to engage in mobile work. From the practical perspective, organizations' understanding of employees' continuance intention in the mobile work context are crucial to achieving desirable ROI in mobile work technologies and practices. Our findings demonstrate that mobile work continuance intention is affected by a myriad of factors including ex post expectation, satisfaction, institutional and individual attributes. As perceived usefulness is influenced by performance, it shows that to enhance users' ex post expectation of mobile work, organizations should not only provide their employees with the necessary technologies but ensure that the technologies are utilized in an effective way that would lead to performance improvements. The experiential constructs in the research model offer organizations actionable recommendations about how to ensure that users' mobile work experience meet or exceed their pre-usage expectation. As mobile workers rely heavily on IT to perform their work, providing mobile workers with superior and timely device and technology support is paramount to the confirmation of expectation. In addition, confirmation of users' expectation can also be achieve by reducing the two primary concerns germane to mobile work that users may have, data security and work life balance concerns. Our study found that satisfaction is central to mobile work continuance; therefore, evaluating mobile workers satisfaction routinely would help organizations gauge mobile worker continuance intention and proactively address any problems detected.

\section{Limitations}

This study has a number of limitations. First, the sample of this study are Chinese knowledge workers, hence the findings may not generalize to other nations or cultures. While cross-cultural validation of the model is beyond the scope of this study, readers should interpret or adopt the findings of this study in other national settings with caution. Second, there may be other antecedents to mobile work continuance intention that were not examined in the current study. For example, task-technology fit research (e.g. [24, 40]) suggests that correspondence between IS functionality and task requirements lead to increased utilization of IS and performance. Other studies have found that subjective norm, which is defined as the normative influences from peers and people who are important to the individual, helped develop the individual's continuance intention [7]. Furthermore, individual factors such as personal innovativeness [36] and computer self-efficacy [49] may play a role in determining mobile work continuance intention. Future research may investigate additional antecedents and the moderating effect of individual factors on the research model of this study. Finally, the underlying assumption of this study is that individuals' behaviors are consistent with their conscious decisions regarding the behaviors, in this case, continuance to engage in mobile work. There are studies that challenged this assumption by suggesting that some continuance behaviors are habitual rather than planned actions [7, 37]. Therefore, in the future, the longitudinal study approach will be valuable to measure both continuance intention and subsequent behaviors more accurately to understand the impact of the antecedents examined in this study on mobile work continuance behaviors.

\section{References}

[1] Ajzen, I., "The theory of planned behavior," Organizational Behavior \& Human Decision Processes (50)2, 1991, pp. 179-211.

[2] Anderson, J., and D. Gerbing, "Structural equation modeling in practice: A review and recommended two-step approach," Psychological Bulletin, (103)3, 1988, pp. 411423.

[3] Bagozzi, R.P., and Y. Yi , "On the evaluation of structural equation models," Journal Academy of Marketing Science (16)1, 1988, pp. 74-94. 
[4] Basole, R.C., "Enterprise mobility: Researching a new paradigm," Information Knowledge Systems Management (7), 2008, pp. 1-7.

[5] Bhattacherjee, A., "Understanding information systems continuance: An expectation-confirmation model," MIS Quarterly (25)3, 2001, pp. 351-370.

[6] Bhattacherjee, A., "An empirical analysis of the antecedents of electronic commerce service continuance," Decision Support Systems (32), 2001, pp. 201-214.

[7] Bhattacherjee, A., and C.P. Lin, "A unified model of IT continuance," European Journal of Information Systems (24)4, 2015, pp. 364-373.

[8] Byrne, B.M., Structural Equation Modeling with LISREL, PRELIS, and SIMPLIS: Basic Concepts, Applications, and Programming. Mahwah, NJ: Lawrence Erlbaum Associates, Inc., Publishers, 1988.

[9] Chen, L., and C. Corritore, "A theoretical model of nomadic culture: Assumptions, values, artifacts and the impact on employee job satisfaction," Communications of the AIS (22), 2008, pp. 235-260.

[10] Chen, L., T. Meservy, and M. Gillenson, "Understanding information systems continuance for information-oriented mobile applications," Communications of the AIS (30), 2012, Article 9.

[11] Chen, L., and R. Nath, "An empirical examination of the impact of wireless local area networks on organizational users," Journal of Electronic Commerce in Organizations (4)2, 2006, pp. 62-81.

[12] Chen, L., and R. Nath, "Impediments to mobile work: An empirical study," International Journal of Mobile Communications (9)5, 2011, pp. 522-540.

[13] Chesley, N., "Blurring boundaries? Linking technology use, spillover, individual distress, and family satisfaction," Journal of Marriage and Family (67)5, 2005, pp. 1237-1248.

[14] Conlin, M., Smashing the clock. Businessweek, December 11, 2006, pp. 60-68.

[15] Crowe, R., and C. Middleton, "Women, smartphones and the work-place: Pragmatic realities and performative identities," Feminist Media Studies (12)4, 2012, pp. 560-569.

[16] Davis, F.D., R.P. Bagozzi, and P.R. Warshaw, "User acceptance of computer technology: A comparison of two theoretical models," Management Science (35)8, 1989, pp. 982-1003.

[17] Davis, G.B., "Anytime/Anyplace computing and the future of knowledge work," Communications of the ACM (42)12, 2002, pp. 67-73.

[18] Dery, K., D. Kolb, and J. Maccormick, "Working with connective flow: How smartphone use is evolving in practice," European Journal of Information Systems (23)5, 2014, pp. 558-570.

[19] Drew, M., "Bringing enterprise mobility to industry," Manufacturers' Monthly, December, 2006, p. 28.

[20] Etezadi-Amoli, J., and A.J. Farhoomand, "On end-user computing satisfaction,” MIS Quarterly, (15)1, 1991, pp. 14.

[21] Ernest-Jones, T., "Pinning down a security policy for mobile data," Network Security (6), 2006, pp. 8-12.

[22] Fishbein, M., and I. Ajzen, Belief Attitude, Intention and Behavior. Reading, MA: Addison-Wesley, 1975.

[23] Fornell, C., and D. Larcker, "Evaluating structural equation models with unobservable variables and measurement error," Journal of Marketing Research (18)1, 1981, pp. 39-50.

[24] Goodhue, D.L., and R.L. Thompson, "TaskTechnology Fit and Individual Performance," MIS Quarterly (19)2, 1995, pp. 213-236.

[25] Gordon, G.E., "Corporate hiring practices for telecommuting homeworkers," In K.E. Christensen (Ed), The New Era of Home-Based Work (pp. 65-78), Boulder and London: Westview Press, 1988.

[26] Hair, J.F., R.E. Anderson, R.L. Tatham, and W.C. Black, Multivariate Data Analysis with Readings. Upper Saddle River, NJ: Prentice-Hall, 1998.

[27] Hoang, A.T., R.C. Nickerson, P. Beckman, and J. Eng, "Telecommuting and Corporate Culture: Implications for the Mobile Enterprise," Information-Knowledge-Systems Management - Enterprise Mobility: Applications, Technologies and Strategies (7)1-2, 2008, pp. 77-97.

[28] Jacobs, G., "Diagnosing the distance: managing communication with dispersed technical workforces," Corporate Communications (9)2, 2004, pp. 118-127.

[29] Jarvenpaa, S.L., K.R. Lang, and V.K. Tuunainen, "Friend of foe? The ambivalent relationship between mobile technology and its users," In Designing Ubiquitous Information Environment: Socio-Technical Issues and Challenges (Sorensen, C., Yoo, Y., Lyytinen, K., and DeGross, J., Eds), (pp. 29-42), Springer, New York, 2005.

[30] Jessup, L.M., D. Robey, "The relevance of social issues in ubiquitous computing environment," Communications of the ACM (45)12, 2002, pp. 88-91.

[31] Karahanna, E., and D.W. Straub, "The psychological origins of perceived usefulness and ease-of-use," Information \& Management (35)4, 1999, pp. 237-250.

[32] Karanasios, S., and D. Allen, "Mobile technology in mobile work: Contradictions and congruencies in activity 
systems," European Journal of Information Systems (23), 2014, pp. 529-542.

[33] Kim, B., and I. Han, "What drives the adoption of mobile data services? An approach from a value perspective," Journal of Information Technology (24)1, 2009, pp. 35-45.

[34] Kim, H.J., H. Choi, and J. Kim, “A comparative study of the effects of low and high uncertainty avoidance on continuance behavior," Journal of Global Information Management (18)2, 2010, pp. 1-29.

[35] Leclercq-Vandelannoitte, A., H. Isaac, and M. Kalika, "Mobile information systems and organizational control: Beyond the panopticon metaphor?" European Journal of Information Systems (23), 2014, pp. 543-557.

[36] Lewis, W., R. Agarwal, V. Sambamurthy, "Sources of influence on beliefs about information technology use: An empirical study of knowledge workers," MIS Quarterly (27)4, 2003, pp. 657-678.

[37] Limayem, M., S. Hirt, and C. Cheung, "How habit limits the predictive power of intention: The case of information systems continuance," MIS Quarterly (31)4, 2007, pp. 705-737.

[38] Lopez-Nicolas, C., F.J. Molina-Castillo, and H. Bouwman, "An assessment of advanced mobile services acceptance: contributions from TAM and diffusion theory models," Information \& Management (45)6, 2008, pp. 359364.

[39] MacCallum, R.C., M.W. Browne, and H.W. Sugawara, "Power analysis and determination of sample size for covariance structure modeling," Psychological Methods (1), 1996, pp. 130-149.

[40] Mathieson, K., and M. Keil, M., "Beyond the interface: Ease of use and task-technology fit," Information \& Management (34)4, 1998, pp. 221-230.

[41] McIntosh, J.C., \& Baron, J.P., Mobile Commerce's Impact on Today's Workforce. International Journal of Mobile Communications, 3(2), 2005, pp. 99-113.

[42] Middleton, C.A., and W. Cukier, "Is mobile email functional or dysfunctional? Two perspectives on mobile email usage," European Journal of Information Systems (15)3, 2006, pp. 252-260.

[43] Middleton, C., R. Scheepers, and V.K. Tuunainen, "When mobile is the norm: researching mobile information systems and mobility as post-adoption phenomena," European Journal of Information Systems (23), 2014, pp. 503-512.

[44] Olson, M.H., "New information technology and organizational culture," MIS Quarterly (6)4, 1982, pp. 71-92.

[45] Picoto, W.N., F. Belanger, and A. Palma-dos-Reis, "An organizational perspective on m-business: usage factors and value determination," European Journal of Information Systems (23), 2014, pp. 571-592.

[46] Popescu, A., "Continuing training in Romania: Reasons and benefits from the perspective of the knowledge economy," Economics \& Sociology (5)2, 2012, pp. 35-49.

[47] Prasopoulou, E., A. Pouloudi, and N. Panteli, "Enacting new temporal boundaries: the role of mobile phones," European Journal of Information Systems (15)3, 2006, pp. 277-284.

[48] Sheppard, B.H., J. Hartwick, and P. Warshaw, "The theory of reasoned action: A meta-analysis of past research with recommendations for modifications and future research," Journal of Consumer Research (15)3, 1988, pp. 325-343.

[49] Thatcher, J. and P. Perrewe, "An empirical examination of individual traits as antecedents to computer anxiety and computer self-efficacy," MIS Quarterly (26)4, 2002, pp. 381-396.

[50] VARBusiness, "Mobile users pursue risky business," VARBusiness, (22)22, 2006, p. 51.

[51] Venkatesh, V., M. G. Morris, G. B. Davis, and F. D. Davis, "User Acceptance of Information Technology: Toward a Unified View," MIS Quarterly (27)3, 2003, pp. 425-478.

[52] Yuan, Y., N. Archer, C.E. Connelly, and W. Zheng, "Identifying the ideal fit between mobile work and mobile work support," Information \& Management (47)3, 2010, pp. 125-137. 